\title{
ESTUDO PRELIMINAR SOBRE A UTILIDADE DA TONOMETRIA GÁSTRICA DURANTE A RETIRADA DA VENTILAÇÃO PULMONAR MECÂNICA EM CRIANÇAS
}

Renato lopez de Souza*, Werther Brunow de Carvalho

Trabalho realizado na Unidade de Cuidados Intensivos Pediátrica, Hospital São Paulo-Escola Paulista de MedicinaUniversidade Federal de São Paulo. SãoPaulo-Brasil.

RESUMO - OBjETIvo. A medida do pH intramucoso (pHi) está sendo estudada e avaliada como um método minimamente invasivo capaz de fornecer informações sobre a oxigenação tecidual, além de apresentar como principal vantagem uma medida regional e não sistêmica, num local com alterações precoces de fluxo sangüíneo mesmo nos estados compensados do choque, como o trato gastrointestinal. A retirada da ventilação pulmonar mecânica pode ocasionar alteração do débito cardíaco, assim como provocar uma redistribuição do fluxo sangǘneo para a musculatura respiratória. 0 objetivo do nosso estudo foi testar a hipótese de ocorrer diminuição do fluxo esplâncnico, detectado pela redução do pHi, após extubação dos pacientes e verificar a possibilidade do $\mathrm{pHi}$ ser um bom índice preditivo para a retirada da ventilação pulmonar mecânica.

Métodos. Estudo clínico prospectivo, não randomizado, não controlado, realizado na unidade de cuidados intensivos de hospital universitário. Avaliação do pHi (gástrico), com um tonômetro com solução salina, em 15 crianças no pós-operatório de cirurgia cardíaca eletiva com circulação extra-corpórea, realizadas medidas I hora antes e após extubação. Foi feita divisão dos pacientes em dois grupos com $\mathrm{pHi} \leq$ ou $>7,32$. Utilizado para análise estatística 0 Teste de Wilcoxon.
Resultados. Dez pacientes (66,7\%) apresentaram queda do pHi após extubação. A média geral do pHi antes da extubação foi de 7,32 $\pm 0,10$ e pós-extubação foi de $7,27 \pm 0,10(p=0,096)$. No grupo com $\mathrm{pHi} \leq 7,32$ e $>7,32$ as médias foram 7,25 $\pm 0,05$ para $7,23 \pm 0,10(p=0,72)$ e $7,4 I \pm 0,07$ para $7,3 I \pm 0,10$ $(p=0,059)$, antes e após extubação, respectivamente. Nenhum paciente apresentou complicações devido ao procedimento, não tivemos nenhum óbito ou desenvolvimento de síndrome da disfunção de múltiplos orgãos. Todos os pacientes foram extubados com sucesso.

Conclusöos. Nossos resultados mostram uma diminuição do pHi confirmando uma redução do fluxo esplâncnico após extubação, entretanto, apesar desta alteração circulatória, os pacientes foram retirados da ventilação pulmonar mecânica e extubados com sucesso. Em nossa amostra o pHi não se mostrou um bom índice preditivo para o desmame. A monitorização do pHi parece ser útil e de fácil execução, porém necessita maiores estudos para avaliar a sensibilidade do método.

UnITERMOs: pH intramucoso. Circulação esplâncnica. Ventilação mecânica e cirurgia cardíaca.
INTRODUÇÃO

A medida do $\mathrm{pH}$ intramucoso do trato gastrointestinal ( $\mathrm{pHi}$ ) está sendo avaliada como método minimamente invasivo capaz de fornecer informação da oxigenação tecidual em circunstâncias onde o paciente não

*Correspondência:
Renato Lopes de Souza
Praça Louveira, 12 - São Paulo-SP
CEP: 03080-050
Tel: (011) 99822746 - Fax: (011) 2942043

apresenta sinais clínicos de instabilidade cardiocirculatória. Parece que nos pacientes nos quais o déficit de oxigênio sistêmico já é evidenciado pela monitorização convencional (hipoperfusão evidente, necessitando vasopressores), não existe um grande benefício da monitorização do pHi. Ela é mais útil naqueles pacientes nos quais as medidas fisiológicas ainda estão dentro da faixa de normalidade, como nos estados compensados do choque. A pesquisa entre as pressões parciais de
$\mathrm{O}_{2} / \mathrm{CO}_{2}$ e o fluxo sangüíneo de orgãos ou tecidos através tonometria começou em 1964 com Bergofsky'. Este autor investigou o equilíbrio da tensão destes gases, em meio líquido, ao redor dos tecidos. Observou o equilíbrio de $\mathrm{O}_{2}$ e $\mathrm{CO}_{2}$ com o líquido instilado na bexiga ou vesícula biliar. Umano após este método é aplicado no jejuno e íleo, mostrando que este equilíbrio também ocorre e era atingido em 30 minutos $^{2}$. Posteriormente, foi desenvolvido o método da tonometria 
que começa a ser utilizado como medida estimada do pH intramucoso, assumindo que a concentração de bicarbonato tecidual é similar ao bicarbonato arterial, assim como o $\mathrm{pCO}_{2}$ do tonômetro é semelhante ao $\mathrm{pCO}_{2}$ da mucosa, e substituindo estes valores na equação de Henderson-Hasselbalch ${ }^{3}$. Mais recentemente, esta técnica foi validada num estudo em animais (porcos) onde foi comparado o pHi através de medidas tonometricamente estimadas com medidas através de microeletrodos intramurais, num grupo controle, um grupo com acidose intestinal por oclusão total de artéria mesentérica superior, um grupo com acidose intestinal por oclusão parcial de artéria mesentérica superior e outro com acidose intestinal induzida por endotoxemia 4 .

A técnica de mensuração do pH intramucoso (gástrico ou intestinal) parece ser capaz de monitorizar estas alterações metabólicas regionais. Esta técnica é de fácil execução, requer apenas a passagem de uma sonda gástrica (o que na maioria dos pacientes é necessário), utiliza uma víscera oca de fácil acesso com proximidade da mucosa (onde existe um sistema de arteríolas e vênulas suceptível à redução do fluxo sangüíneo e redução da oferta de oxigênio); sem necessidade de quebra da barreira de pele e com raríssimas complicações ${ }^{5}$.

A medida do $\mathrm{pH}$ intramucoso representa a concentração de íons hidrogênio na mucosa, valores estes que dependem do metabolismo do tecido. Este pH é bem diferente do $\mathrm{pH}$ intraluminal, o pH intramucoso considerado normal é $\geq 7.35^{6}$. A maioria dos trabalhos considera o $\mathrm{pHinor-}$ mal igual a 7,38 $\pm 0,03$, considerando desta forma um pHi baixo $\leq 7,32$ (dois desvios padrão para baixo). Esta explicação não é muito consistente, porém estes valores são usados para definir pHi normal ou anormal ${ }^{7}$. Na pediatria o $\mathrm{pHi}$ é semelhante ao do adulto, a medida do pHi em crianças saudáveis, normoventiladas e hemodi- nâmicamente estáveis mostrou valores em média de 7,34 0 0,027 com limites de 7,29 a $7,46^{8}$.

As vantagens deste método são fácil execução e o baixo custo. Esta medida regional num local como o estômago ou intestino é de grande valia, pois como comentado anteriormente, nos estados de choque ou hipoxia, estas alterações ocorrem precocemente, enquanto que em outros locais (por mecanismos compensátorios) pode ocorrer manutenção ou até aumento do fluxo sangüíneo.

A tonometria tem sido usada e validada como método diagnóstico nos estados de hipovolemia (hemorragias ou choque) e o pHi, assim como outras medidas diretas de fluxo sangüíneo, mostraram alta sensibilidade na determinação da efetividade da reposição volêmica no tratamento do choque hemorrágico?. O pHi tem sido usado como índice preditivo de mortalidade e desenvolvimento de disfunção de múltiplos órgãos ${ }^{10-12}$. Entretanto, alguns autores acham que esta medida não é suficientemente sensível para pequenas e rápidas alterações da função circulatória, portanto não sendo úteis nas tomadas de decisões para titulação terapêutica ${ }^{13}$.

\section{Objetivos}

O objetivo de nosso estudo foi testar a hipótese de ocorrer diminuição de fluxo sangüíneo esplâncnico detectado pela redução do pHiapós extubação dos paciente, assim como avaliar o insucesso do procedimento nos pacientes e necessidade de re-intubação.

\section{Métodos}

Estudo prospectivo, não randomizado, realizado em UCI Pediátrica, avaliando os pacientes em pós-operatório de correção eletiva de cardiopatia congênita com circulação extracorpórea.
Este protocolo foi apresentado à Comissão de Ética do hospital que aprovou sua realização. Antes do procedimento, os pais ou responsáveis pelo paciente foram devidamente informados e consentiram em participar do estudo por escrito, tendo liberdade de, a qualquer momento, solicitar a saída do protocolo sem qualquer prejuízo ao atendimento.

\section{População}

Foram estudados 15 pacientes no período pós-operatório que obedeciam os seguintes critérios:

1. Cardiopatia congênita submetidos à cirurgia cardíaca eletiva com circulação extracorpórea.

2. Peso mínimo de 9 kg e máximo de 20 kg. Foi selecionada esta faixa de peso para que houvesse a possibilidade da passagem da sonda de tonometria, sem traumatismo para o paciente.

3. Possibilidade de utilização de sonda nasogástrica. Exclusão de pacientes com qualquer contra indicação de passagem da sonda, como obstrução nasofaríngea ou esofágica, patologia esofágica (divertículos, tumores, perfuração ou estenose), varizes de esôfago, fístula traqueoesofágica ou sangramento gastrointestinal.

\section{Equipamento}

A sonda de tonometria NGS Catheter Trip $^{\mathrm{TM} M}$ (gastric tonometer and sump) é produzida pela Tonometrics, Inc., Worcester, MA, USA. Esta sonda é uma combinação de tonômetro gástrico e sonda de ventilação e sucção. Possui três vias, uma de sucção com adaptador universal, outra via de ventilação e a terceira é a do lumen do tonômetro, que inicia com uma torneira tipo "stopcock" (três vias) e termina no balão siliconizado permeávelao $\mathrm{CO}_{2}$. O comprimento do catéter éde $122 \mathrm{~cm}$ e 16 French $(5,3 \mathrm{~mm})$.

A passagem da sonda (tonômetro) por via oral foi realizada no centro cirúrgico, em condições assépticas após indução da 
SoUzA RL ET AL.

anestesia. As sondas foram previamente verificadas quanto à integridade do balão e completa retirada de ar (o balão de silicone foi preenchido e esvaziado algumas vezes com $4 \mathrm{ml}$ de solução salina, porfim aspirada lentamente até o colapso do balão). Depois de assegurada a localização da sonda no estômago, pela ausculta, foram infundidos $2,5 \mathrm{ml}$ de solução salina $0,9 \%$ (soro fisiológico) no balẫo do tonômetro. $\mathrm{Na}$ $\mathrm{UCl}$ a posição das sondas foi confirmada radiologicamente.

Para a medida do $\mathrm{CO}_{2}$ foi retirada a solução salina utilizando técnica anaeróbica, desprezando I ml da solução que corresponde ao espaço morto da sonda e anotado otempo de equilibrio. A coleta simultânea de gasometria foi realizada através de cateter em artéria radial para monitorização de pressão arterial invasiva. As amostras, tanto da solução salina como do sangue arterial, foram transportadas em caixa de isopor com gelo e processadas de imediato no laboratório. Estas amostras foram analisadas no aparelho de gasometria Radiometer Copenhagen ABL 330.

\section{Medidas}

Os pacientes estavam em ventilação pulmonar mecânica em aparelhos de fluxo contínuo no modo de ventilação mandatória intermitente.

Os pacientes foram avaliados clinicamente e através de outras variáveis quantitativas e qualitativas. As qualitativas foram: alterações do nível de consciência, perfusão periférica, amplitude dos pulsos, gradiente térmico. As quantitativas foram: gasometria arterial e venosa, $\mathrm{SpO}_{2}, \mathrm{FC}$, PAM, PVC, PAE, débito urinário (m//Kg/ hora), lactato, extração de $\mathrm{O}_{2}$, gradiente veno-arterial de $\mathrm{CO}_{2}$.

Todos os pacientes recebiam ranitidina na dose de $3 \mathrm{mg} / \mathrm{kg} / \mathrm{dia}$ ( para evitaro possível efeito de tamponamento pancreático, resultando em retro-difusão de $\mathrm{CO}_{2}$ gerado pela reação entre o hidrogênio e o bicarbonato) $)^{14}$, recebiam também suporte cardiovascular com dobutamina. Os pacientes apresentavam estabilidade hemodinâmica e condições clínicas e gasométricas para a extubação.

As medidas foram realizadas uma hora antes e uma hora após extubação do paciente e os pacientes foram divididos em dois grupos segundo o pHi antes da extubação, normal $(>7,32)$ ou baixo $(\leq 7,32)$.

A conduta do médico assistente não foi influenciada pelos resultados da tonometria, este não tomou conhecimento dos resultados.

\section{Cálculo do pHi}

Assumindo que a $\mathrm{pCO}_{2}$ intramural entre emequilibrio coma $\mathrm{pCO}_{2}$ intraluminal eque o bicarbonato intramural é semelhante ao bicarbonato arterial, o cálculo do pH intramucoso (pHi)foifeito utilizando a medida da $\mathrm{pCO}_{2}$ da solução salina $\left(\mathrm{pCO}_{2}\right.$ ss) e o bicarbonato arterial $\left(\mathrm{HCO}_{3}\right.$ art.) na equação de Henderson-Hasselbalch. $\mathrm{pHi}=6.1+\log$ $\left[\mathrm{HCO}_{3}\right.$ art $] /\left[\mathrm{pCO}_{2} \mathrm{ss} \times 0,03 \mathrm{l}\right.$ ] Onde 6, I é o pK para o sistema $\mathrm{HCO}_{3} / \mathrm{CO}_{2}$ no plasma a $37^{\circ} \mathrm{C} ; \mathrm{HCO}_{3}$ éa concentração de bicarbonato ( $\mathrm{mM} / \mathrm{L}$ ) do sangue arterial; $\mathrm{PCO}_{2}$ s é a $\mathrm{pCO}_{2}$ ajustada pelo tempo de equilíbrio da soluçã̃o salina do tonômetro e 0,03 I é a solubilidade do $\mathrm{CO}_{2}$ no plasma $[(\mathrm{mM} / \mathrm{L}) /$ $\mathrm{mmHg}$ a $37^{\circ} \mathrm{C}$.

As coletas foram feitas após um tempo de equilíbrio entre o tempo de infusão da solução salina e sua retirada. Foi utilizado de acordo com o tempo de equilíbrio um fator de correção para o $\mathrm{pCO}_{2}$ (determinado in vitro) estipulado pelo fabricante.

\section{Análise estatística}

Foram comparados os valores de $\mathrm{pHi}$ antes e após extubação dos pacientes, diminuição do pHi após extubação, assim como a comparação dos dois grupos com pHi normal ou baixo, para identificar as possibilidades de insucesso na retirada da ventilação pulmonar mecânica.
As variáveis numéricas foram representadas por média, desvio padrão, mediana, valores mínimo e máximo. As medidas pré e pós-extubação foram comparadas pelo Teste de Wilcoxon.

Adotou-se o nível de significância de $0,05(\alpha=5 \%)$. Níveis descritivos (p) inferiores a esse valor foram considerados significantes.

\section{Resultados}

As características da população segundo idade, peso e estatura, assim como tempo médio de internação estão na Tabela I.

Dez pacientes $(66,7 \%)$ apresentaram queda do pHi após extubação, como mostrado na Tabela 2.

A média do pHi antes da extubação foi de 7,32 $\pm 0,10$ e pós-extubação foi de $7,27 \pm 0,10$. Houve uma diminuição do pHi após extubação e a diferença destas médias não foi estatisticamente significante $(p=0,096)$. (Tabela 3).

Os pacientes foram divididos em dois grupos, aqueles que apresentavam $\mathrm{pHi}$ normal $(>7,32)$ e aqueles que apresentavam pHi baixo $(\leq 7,32)$ antes da extubação.

No primeiro grupo, com sete pacientes a média do $\mathrm{pHi}$ foi de $7,4 \mathrm{I} \pm 0,07$ préextubação e de 7,31 $\pm 0,10$ pós-extubação $(p=0,0592)$. No segundo grupo, com oito pacientes, a média do pHi antes da extubação foi de 7,25 $\pm 0,05$ e pós-extubação de $7,23 \pm 0,10$, também mostrando uma diminuição do pHi, porém sem diferença estatisticamente significante $(p=0,726)$. (Tabela 4).

Os pacientes apresentavam estabilidade hemodinâmica e condições clínicas e gasométricas para a extubação.

Nenhum paciente apresentou complicações devido ao procedimento, não tivemos nenhum óbito ou desenvolvimento de síndrome da disfunção de múltiplos órgãos. Todos os pacientes foram extubados com sucesso. 


\begin{tabular}{lc}
\hline \multicolumn{2}{c}{ Tabela I - Características da população } \\
\hline Variável & Média \pm D.P. \\
Idade(anos/meses) & $3 a 8 \mathrm{~m} \pm 2 \mathrm{a} 5 \mathrm{~m}$ \\
Peso $(\mathrm{kg})$ & $12,30 \pm 3,05$ \\
Estatura(cm) & $93,60 \pm 14,04$ \\
Tempode internaçãonaUCl(dias) & $2,5 \pm 1,0$ \\
\hline
\end{tabular}

\begin{tabular}{|c|c|c|c|c|}
\hline PACIENTES & PATOLOGIAS & CIRURGIAS & $\begin{array}{c}\text { PRÉ- } \\
\text { EXTUBAÇÃO }\end{array}$ & $\begin{array}{c}\text { PÓS- } \\
\text { EXTUBAÇÃO }\end{array}$ \\
\hline । & $\begin{array}{l}\text { Insuficiênciamitral gravee } \\
\text { HP }\end{array}$ & Plastiade mitral & 7,41 & 7,42 \\
\hline 2 & Insuficiênciamitral grave & Plastia demitral & 7,26 & 7,19 \\
\hline 3 & $\begin{array}{l}\text { DuplaviadeentradadeVE } \\
\text { ebandagem depulmonar }\end{array}$ & DerivaçãoCavo-pulmonar & 7,36 & 7,35 \\
\hline 4 & $\begin{array}{l}\text { Duplaviade saída de VEe } \\
\text { estenosede pulmonar }\end{array}$ & Correçãototal & 7,44 & 7,44 \\
\hline 5 & $\begin{array}{l}\text { Dupla via de saída de VD e } \\
\text { estenosede pulmonar }\end{array}$ & $\begin{array}{l}\text { Atriosseptectomiae "shunt" } \\
\text { central aorto-pulmonar }\end{array}$ & 7,31 & 7,17 \\
\hline 6 & $\begin{array}{l}\text { Origemanômalade } \\
\text { coronaria esquerda e } \\
\text { insuficiênciamitral }\end{array}$ & $\begin{array}{l}\text { Reimplantedecoronaria } \\
\text { esquerda }\end{array}$ & 7,55 & 7,29 \\
\hline 7 & $\begin{array}{l}\text { Tetralogiade Fallot } \\
\text { (corrigida) }\end{array}$ & Fechamento de CIV residual & 7,24 & 7,40 \\
\hline 8 & $\begin{array}{l}\text { Tetralogiade Fallote } \\
\text { Blalock-Taussing }\end{array}$ & $\begin{array}{l}\text { Correçãototal efechamento } \\
\text { doB.Taussing }\end{array}$ & 7,36 & 7,28 \\
\hline 9 & Insuficiênciamitral residual & Trocadeválvula mitral & 7,22 & 7,33 \\
\hline 10 & ClVeHP & FechamentodaCIV & 7,21 & 7,28 \\
\hline$\|$ & CIVeHP & FechamentodaCIV & 7,18 & 7,11 \\
\hline 12 & $\begin{array}{l}\text { Atresia detricúspidee CIA } \\
\text { eCIV }\end{array}$ & $\begin{array}{l}\text { Ampliação ClAe } \\
\text { anastomoseveiacava } \\
\text { superior etronco da pulmonar }\end{array}$ & 7,30 & 7,23 \\
\hline B & CIVeHP & FechamentodaCIV & 7,38 & 7,25 \\
\hline 14 & CIVsubaórtica & FechamentodaCIV & 7,37 & 7,17 \\
\hline 15 & Tetralogiade Fallot & $\begin{array}{l}\text { Correç̧ãototal eampliação } \\
\text { devia de sáddade VD }\end{array}$ & 7,28 & 7,15 \\
\hline
\end{tabular}

VE-ventrículo esquerdo, VD - ventrículo direito, HP - hipertensão pulmonar, CIV - comunicaçãointerventricular, CIA-comunicação interatrial. 
SOUZA RL ET AL.

Tabela 3 - Valores do pHi antes e após extubação (I5 pacientes)

\begin{tabular}{cc}
\hline & pHi \\
& Média \pm D.P. \\
Pré-extubação & $7,32 \pm 0,10$ \\
Pós-extubação & $7,27 \pm 0,10$ \\
& $z=1,66 p=0,0962$ \\
\hline
\end{tabular}

Tabela 4 - Pacientes que antes da extubação tinham pHi > 7,32 (7 pacientes) ou $\mathrm{pHi} \leq 7,32$ (8 pacientes)

\begin{tabular}{ccc}
\hline Condição & $\begin{array}{c}\text { pHi }>\text { 7,32 } \\
\text { Média } \pm \text { D.P. }\end{array}$ & $\begin{array}{c}\text { pHi } \leq 7,32 \\
\text { Média } \pm \text { D.P. }\end{array}$ \\
Pré-extubação & $7,41 \pm 0,07$ & $7,25 \pm 0,05$ \\
Pós-extubação & $7,31 \pm 0,10$ & $7,23 \pm 0,10$ \\
& $z=1,89 p=0,059$ & $z=0,35 p=0,726$ \\
\hline
\end{tabular}

\section{Discussão}

As interações cardiorrespiratórias provocam repercussões clínicas nos pacientes em ventilação pulmonar mecânica (VPM), principalmente nos cardiopatas. Aretirada da VPM pode alterar o débito cardíaco, assim como provocar uma redistribuição do fluxo sangüíneo aumentando o fluxo na musculatura respiratória e no consumo de oxigênio com prejuízo em outros territórios, por vezes provocando insucesso na retirada da VPM nos pacientes com instabilidade hemodinâmica. A tonometria gástrica poderia ser um indicador para avaliar esta condição.

Em nosso estudo, os pacientes apresentavam condições clínicas e gasométricas para retirada da VPM, entretanto, mesmo sem evidenciarem alterações do débito cardíaco, da pressão arterial, de gradiente térmico, ou até mesmo acidose metabólica, eles apresentaram uma redução do $\mathrm{pH}$ intramucoso para níveis de 7,27 em média. Esta queda no pHi pode representar uma diminuição do fluxo esplâncnico após a retirada da VPM, como demonstrado no trabalho de Elizalde et al. que investigaram a hipótese que a hipoperfusão da mucosa gástrica poderia ter um papel importante no desenvolvimento da acidose intramucosa nos pacientes em $V_{P M}{ }^{15}$. Neste estudo foram avaliados 17 pacientes e divididos em dois grupos com pHi baixo $(\leq 7,32)$ e pHi normal $(>7,32)$. O fluxo sangüíneo foi avaliado através de dois métodos (fluxometria com Laser-doppler e espectrofotometria de reflectância) e comparados com o pHi . O principal dado deste estudo foi que a acidose intramucosa nestes pacientes está associada à redução da perfusão da mucosa gástrica. Isto poderia dificultar a retirada da ventilação devido à instabilidade cardiocirculatória no pós-operatório de cirurgias cardíacas.

Muitos critérios têm sido utilizados para predizer o sucesso da retirada da VPM. Thiagarajan et al. realizaram um estudo prospectivo com 227 crianças em VPM com o objetivo de verificar quais os fatores associados ao sucesso na extubação. Variáveis de mecânica respiratória foram utilizadas e conclui-se que os melhores índices seriam a complacência, resistência, oxigenação e pressão, e também que a falha na extubação estava ligada à disfunção do sistema respiratório ${ }^{16}$.

A isquemia da mucosa gástrica pode funcionar como um dado indireto de insucesso desta retirada, uma vez que a diminuição do fluxo sangüíneo esplâncnico e a acidose intramural podem ocorrer devido ao desvio de fluxo de sangue para satisfazer a demanda da musculatura respiratória. Mohsenifar et al. mostraram que os pacientes que não tiveram sucesso na retirada da VPM tinham pHi mais baixos ${ }^{17}$. Estes autores observaram em seu trabalho que 0 grupo de pacientes que apresentaram queda do pHi durante o "desmame" da VPM (7,36 durante VPM para 7,09 durante 0 "desmame", $p<0,0$ I) tiveram falha na retirada da VPM. Neste trabalho a tonometria teve uma sensibilidade e especificidade de 100\% em predizer o sucesso ou a falha na retirada da VPM, sendo um sinal precoce para identificar a possibilidade de insucesso. Bouachour etal. também obtiveram bons resultados com a utilização da tonometria durante o desmame; na análise de 26 pacientes adultos com DPOC, este índice mostrou 100\% de especificidade e sensibilidade em predizer falha no desmame ${ }^{18}$. Outros autores acreditam que as mudanças do $\mathrm{pHi}$ parecem estar relacionadas principalmente às variações da $\mathrm{pCO}_{2}$ arterial, e que a diminuição do fluxo sangüíneo da mucosa gástrica é devido à diferença de pressão transdiafragmática ${ }^{19}$. Maldonado et al. observaram as mudanças da $\mathrm{pCO}_{2}$ gástrica durante o desmame e relatou um aumento significativo quando houve insucesso e redução nos pacientes que foram desmamados com sucesso; entretanto, neste estudo o melhor preditor foi a relação FRNC, concluindo que a tonoOmetria não melhora as características operacionais do desmame ${ }^{20}$. 
Natentativa de identificar os pacientes de risco de insucesso na retirada da VPM e com a dificuldade de trabalharmos com uma amostra pequena, observamos uma diminuição do fluxo sangüíneo esplâncnico após extubação, porém, mesmo com esta alteração hemodinâmica, os pacientes tiveram boa evolução. Um dos fatores relevantes para esta condição pode ter sido a utilização de dobutamina, melhorando assim a redistribuição do fluxo sangüíneo através da mucosa gastrointestinal. Todos os pacientes em nosso estudo fizeram uso de dobutamina, que só foi suspensa após a extubação. Das drogas que poderiam atuar na circulaçãao esplâncnica esta é uma das principais, já que existem muitos trabalhos mostrando a melhora do pHi com utilização de dobutamina no pós-operatório de cirurgias cardíacas ${ }^{21-22}$. Isto pode ter causado algum impacto nos resultados, sendo um fator de melhora do pHi (por melhora do débito ou redistribuição de sangue na mucosa gastrointestinal) ocasionando uma recuperação hemodinâmica nos pacientes deste estudo.

Outra possibilidade de insucesso seria naqueles pacientesquejáapresentavam pHibaixo antes do procedimento, então estratificamos os pacientes em dois grupos. No primeiro grupo compHinormal antes da extubaçãoe no outro grupo, que teria maior risco de insucesso de extubação, com pHi baixo antes do procedimento, entretanto em nenhum dos grupos houve retorno à VPM. Estes achados foram semelhantes ao trabalho de lbiza et al. que testaram a hipótese da tonometria gástrica como sinal precoce de falha no "desmame" da VPM em 24 pacientes pedíátricos e concluíram que não houve diferença estatisticamente significante do $p$ Hientre os pacientes queforam retirados com sucesso da ventilação e aqueles que retornaram à VPM ${ }^{23}$. Os autores atribuem estes resultados talvez ao tamanho da amostra e ao fato de que a tonometria não pode avaliar problemas de vias aéreas superiores (maior causa de insucesso na retirada da ventilação).
Um fator importante foi que nossos pacientes não tinham patologia pulmonar, sendo pacientes "ideais" para retirada da VPM após estabilização pós-operatória. É provável que o $\mathrm{pHi}$ possua maior valor, em termos de sensibilidade e especificidade, em pacientes graves com alterações pulmonares e/ou cardiocirculatórias (como sepse ou Síndrome de desconforto respiratório agudo) onde qualquer condição que leva à disóxia ou diminuição da reserva energética possa influenciar na retirada da VPM.

Há muito é conhecido que a isquemia intestinal pode ser uma causa importante do desenvolvimento de disfunção de múttiplos órgãos eóbito nos pacientes graves ${ }^{24}$. Vários trabalhos apontam o pHi como uma variável de grande sensibilidade e especificidade em predizer complicações e mortalidade, entretanto nossos pacientes tiveram boa evolução. Mythen eWebb relataram umaumento do número de complicações em cirurgias eletivas e aumento dos custos hospitalares em pacientes com pHi gástrico baixo no intra-operatório ${ }^{25}$. Neste estudo com 51 pacientes, 14 desenvolveram complicações (7 com disfunção de múltiplos órgãos) e seis pacientes faleceram.

Num estudo semelhante ao nosso, Rey et al. analisando o pHi em crianças no pós operatório de cirurgia cardíaca observaram que a diminuição do pHi precedia as complicações hemodinâmicas nestes pacientes ${ }^{26}$. Casado-Flores et al., num estudo observando 5 I crianças, verificaram maior mortalidade nos pacientes com pHi menor que 7,30 na admissão, entretanto, não houve relação do pHicom risco maior de desenvolvimento de disfunção de múltiplos orgãos. ${ }^{27}$

Nos pacientes em VPM os valores baixos de pHi, mesmo com estabilidade hemodinâmica, podem significar maior mortalidade, como mostrado por Moshenifar et al. ${ }^{28}$, que encontraram uma mortalidade de $66 \%$ num grupo de pacientes com $\mathrm{pHi}<7,25$ (com epecificidade de $86 \%$ e sensibilidade de $83 \%$ em predizer mortalidade). A comparação com os trabalhos realizados por Moshenifar fica dificil uma vez que este utiliza em seus estudos a dosagem do $\mathrm{CO}_{2}$ no suco gástrico (aspirado diretamente e analisado) e não a tonometria com a medida do $\mathrm{CO}_{2}$ na solução salina, ou mais recentemente, ar contido num balão siliconizado ${ }^{29}$.

Não observamos nenhuma relação do pHi com o insucesso na retirada da VPM, dez (66,7\%) pacientes apresentaram queda do pHi após a extubação e nenhum deles necessitou re-intubação. Isto talvez tenha ocorrido por que os pacientes conseguiram, através de adaptações fisiológicas, estabilizar a situação ou a tonometria não é um bom método para avaliar possibilidade de "desmame" da VPM e insucesso na extubação neste tipo de pacientes. A monitorização do pHi parece ser útil e de fácil execução, porém são necessários maiores estudos para determinar a sensibilidade do método.

\section{SUMMARY}

Preliminary study about gastric toNOMETRY USEFULNESS, DURING WEANING FROM MECHANICAL VENTILATION IN CHILDREN

OBIECTNE. The intramucosalpHimeasurement has been studied and evaluated as a minimally invasive method able to offer information about the tissue oxygenation. It's most important advantage is to be a regional not systemic measurement in a place with early changes of bloodflow, even in the compensated states of shock, as the gastrointestinal tract.

The weaning from mechanical ventilation may cause cardiac output changes as well as redistribution of blood flow to the respiratory muscles. Our aim was to test the hypothesis that the splanchnic blood flow decreases may occur after patients extubation, detected by pHi reduction, and to verifyiff Hican be a good predictive index for weaning from mechanical ventilation.

Methods. Clinical prospective non-ran- 
SoUza RL ET AL.

domized, uncontrolled study, realized in a University Hospital PICU. Assesment of pHi (gastric) in 15 children in the post-operative of elective cardiac surgery with cardiopulmonary bypass. A tonometer with saline solution was used and measurements were made one hour before and after extubation. The patients were divided into two groups with pHi $\leq$ or > 7.32. The Wilcoxontest was used for statistical analysis.

RESULTS. The pHidecreased after extubation in 10 patients (66.7\%). The overall mean before extubation was $7.32 \pm 0.10$ and after extubation was $7.27 \pm 0.10$ $(p=0.096)$. In the group with $p H i \leq 7.32$ and $>7.32$ the means were $7.25 \pm 0.05$ to $7.32 \pm 0.10(p=0.72)$ and $7.41 \pm 0.07$ to $7.31 \pm 0.10(p=0.059)$, before and after extubation respectively. None of the patients presented complications due to the procedure; no deaths or development of multiple organ failure occurred. All of the patients were successfuly weaned.

CONCLUSIONs. Our results show pHi decreasing confirming splanchnic blood flow reduction after extubation, however in despite of this circulatory change the patients were weaned from mechanical ventilation and successfully extubated. In this trial the pHiwas not a good predictive index. The pHi monitoring seems to be useful and reliable, but it requires further studies to evaluate its sensivity. [RevAssoc Med Bras 2002; 48(I): 66-72]

KEY wORDS: Intramucosal pH. Splanchnic circulation. Mechanical ventilation and cardiac surgery.

\section{REFERÊNCIAS}

I. Bergofsky EH. Determination of tissue $\mathrm{O}_{2}$ tensions by hollow visceral tonometers: effect of breathing enriched $\mathrm{O}_{2}$ mixtures. J Clin Invest 1964: 43:193-200.

2. Dawson AM, Trenchard D, Guz A. Small bowel tonometry: assesment of small gut mucosal oxygen tension in dog and man. Nature 1965; 206: 943-4.

3. Fiddian-Green RG, Pittenger G, Whitehouse $\mathrm{BS} \mathrm{Jr}$. Back-diffusion of $\mathrm{CO}_{2}$ and its influence on the intramural $\mathrm{pH}$ in gastric mucosa. J Surg Res 1982; 33:39-48.

4. Antonsson JB, Boyle III CC, Kruithoff KL, Wang $H L$, Sacristan E, Rothschild HR. Validation of tonometric measurement of gut intramural $\mathrm{pH}$ during endotoxemia and mesenteric occlusion in pigs. Am J Physiol 1990; 259:G519-23.

5. Clark CH, Gutierrez G. Gastric intramucosal $\mathrm{pH}$ : a non invasive method for the indirect measurement of tissue oxygenation. Am J Crit Care 1992; 1:53-60.

6. Gutierrez G. Cellular energy metabolism during hypoxia. Crit Care Med 1991; 19:619-26.

7. Mythen MG, Webb AR. The role of gut mucosal hypoperfusion in the pathogenesis of post-operative organ dysfunction. Intensive Care Med 1994: 20:203-9.

8. Apostolakos MJ. The gastrointestinal tract and critical illness: is more perfusion better? Chest 1995: 108:|49|-2.

9. Nordin A, Mäkisalo H, Mildh L, Höckerstedt $\mathrm{K}$. Gut intramucosal $\mathrm{pH}$ as an early indicator of effectiveness of therapy for hemorrhagic skock. Crit Care Med 1998; 26: I |l0-7.

10. Gutierrez G, Palizas F, Doglio G, Wainsztein N, Gallesio A, Pacin J et al. Gastric intramucosal $\mathrm{pH}$ as a therapeutic index of tissue oxygenation in critically ill patients. Lancet 1992: 339:195-9.

I I. Marik PE. Gastric intramucosal pH. A better predictor of multiorgan dysfunction syndrome and death than oxygen-derived variables in patients with sepsis. Chest 1993; 104:225-9.

12. Maynard N, Bihari D, Beale R, Smithies M, Baldock G, Mason R et al. Assesment of splanchnic oxygenation by gastric tonometry in patients with acute circulatory failure. JAMA 1993; 270: 1203-10.

13. Shoemaker WC. Temporal physiologic patterns of shock and circulatory dysfunction based on early descriptions by invasive and noninvasive monitoring. New Horizons 1996; 4:300-18.

14. Heard SO, Helsmoortel CM, Kent JC, Shahnarian A, Fink MP. Gastric tonometry in healthy volunteers: effect of ranitidine on calculated intramural $\mathrm{pH}$. Crit Care Med 1991: $\quad$ 19:27|-4

I 5. Elizalde JI, Hernández C, Llach J, Monton C, Bordas JM, Pique JM et al. Gastric intramucosal acidosis in mechanically ventilated patients: role of mucosal blood flow. Crit Care Med, 1998; 26:827-32.

16. Thiagarajan RR, Bratton SL, Martin LD, Brogan TU, Taylon D. Predictors of successful extubation in children. Am J Respir Crit Care Med 1999; 160:1562-6.
17. Mohsenifar Z, Hay A, Hay J, Lewis MI, Koerner SK. Gastric intramural $\mathrm{pH}$ as a predictor of success or failure in weaning patients from mechanical ventilation. Ann Intern Med 1993; 119:794-8.

18. Bouachour G, Guiraud J-P, Roy PM, Alquier P. Gastric intramucosal pH: na indicator of weaning outcome from mechanical ventilation in COPD patients. Eur Respir J 1996; 9:1868-73.

19. Bocquillon N, Mathieu D, Neviere R, Lefevre M, Marechal X, Wattel F. Gastric mucosal pH and blood flow during weaning from mechanical ventilation in patients with chronic obstructive pulmonary disease. Am J Respir Crit Care Med 1999; 160:1555-61.

20. Maldonado A, Bauer TT, Ferrer M, Hernandez C, Arancibia F, Rodriguez-Rosin R et al. Capnometric recirculation gas tonometry and weaning from mechanical ventilation. Am J Respir Crit Care Med 2000; 161:171-6.

21. Uusaro A, Ruokonen E, Takala J. Splanchnic oxygen transport after cardiac surgery: evidence for inadequate tissue perfusion after stabilization of hemodynamics. Intensive Care Med 1996; 22:26-33.

22. Silva E, DeBacker D, Créteur J, Vincent J-L. Effects of vasoactive drugs on gastric intramucosal pH. Crit Care Med 1998: 26:1749-58.

23. Ibiza E, Abengochea A, Modesto $V$ et al. Gastric intramural $\mathrm{pH}$ as a predictor of success in weaning pediatric patients from mechanical ventilation. In: 2nd World Congress on Pediatric Intensive Care. Rotterdam, 1996. Abstracts. Intensive Care Med 1996; 22:SI73.

24. Fiddian-Green RG. Splanchnic ischaemia and multiple organ failure in the critically ill. Ann R Coll Surg Engl 1988; 70:128-34.

25. Mythen MG, Webb AR. Intra-operative gut mucosal hypoperfusion is associated with increased post-operative complications and cost. Intensive Care Med 1994; 20:99-104.

26. Rey CC, Tarrio FR, Campaña MB et al. Tonometría gástrica en niños críticamente enfermos: estudio preliminar. An Esp Pediatr 1995: 42:424-8.

27. Casado-Flores J, Mora E, Perez-Corral F, Martinz-Azagra A, Garcia-Teresa MA, RuizLopez MJ. Prognostic value of gastric intramucosal $\mathrm{pH}$ in critically ill children. Crit Care Med 1998; 26: | | 23-7.

28. Mohsenifar Z, Collier J. Koerner SK. Gastric intramural $\mathrm{pH}$ in mechanically ventilated patients. Thorax 1996; 51:606-10.

29. Fielding RE, Baldock G. Development in gastric tonometry. Int Care World 1998; 15: 14 -9.

Artigo recebido: |5//2/2000 Aceito para publicação: | 9/06/200 | 